\title{
THE AUTHORS REPLY
}

Thomas suggests that prevention of melanoma recurrence is neither desirable in nor beneficial to patients. This curious position is inconsistent with a trial for which he was principal investigator, which used loco-regional recurrence as the primary end point. ${ }^{1} \mathrm{He}$ equates outcomes from early treatment of nodal metastases with delayed treatment, which MSLT-I clearly disproves. Early treatment, guided by sentinel-node biopsy, halves the extent of nodal involvement at surgery ${ }^{2}$ and decreases the rate of surgical complications. ${ }^{3}$ Early treatment also results in a clinically and statistically significant decreased risk of death from melanoma among patients with nodal metastases from intermediate-thickness melanomas. His appeal to the NCI actually evoked a clear statement in 2007 from the chief of the Clinical Investigations Branch that the NCI "does not interfere with the peer review process and depends on journal reviewers and editors to fairly referee scientific disagreements" (Abrams J: personal communication). This process was vigorously undertaken by the reviewers and editors of the Journal. Reviewers thought the addition of distant disease-free survival for the entire population (which insignificantly favored the biopsy group [hazard ratio for distant metastasis, $0.89 ; 95 \%$ confidence interval, 0.70 to $1.13 ; \mathrm{P}=0.34]$ ) did not warrant displacement of the presented results.

Thomas's statement that the curves showing the cumulative incidence of nodal metastasis are parallel between 8 and 10 years of follow-up is inaccurate. More than one fourth of the remaining minimal numerical difference between the curves evaporates during those 2 years. The criticism is, in any case, irrelevant since the rates are not statistically different, even with existing follow-up. His repetition of the illogical concept of "prognostic false positivity" therefore remains entirely without merit.

Cady comments that a survival benefit in one subgroup may offset a disadvantage in another. In MSLT-I, a separate analysis involving a prospectively defined subgroup of patients with node-positive disease was justified by a strong biologic rationale. Survival 
among patients in this subgroup who underwent sentinel-node biopsy was substantially improved. Examination of the potentially offsetting group of node-negative patients (Fig. 3C of our article) reveals no suggestion of disadvantage in node-negative patients to counteract this benefit. It is likely that the considerable benefit to node-positive patients is simply diluted to statistical nonsignificance by the preponderance of node-negative patients, who derived prognostic, but not therapeutic, value.

Regarding earlier literature reviews, we draw a different conclusion. Although trials of elective node dissection did not show a statistically significant benefit from early nodal intervention, they consistently showed an advantage to early dissection that approached significance. Lack of statistical significance in underpowered trials should not be considered a demonstration of equality.

\section{References}

1. Thomas JM, Newton-Bishop J, A'Hern R, et al. Excision margins in high-risk malignant melanoma. N Engl J Med. 2004; 350:757-66. [PubMed: 14973217]

2. Morton DL, Thompson JF, Cochran AJ, et al. Sentinel-node biopsy or nodal observation in melanoma. N Engl J Med. 2006; 355:1307-17. [Erratum, N Engl J Med 2006;355:1944.]. [PubMed: 17005948]

3. Faries MB, Thompson JF, Cochran A, et al. The impact on morbidity and length of stay of early versus delayed complete lymphadenectomy in melanoma: results of the Multicenter Selective Lymphadenectomy Trial (I). Ann Surg Oncol. 2010; 17:3324-9. [PubMed: 20614193] 